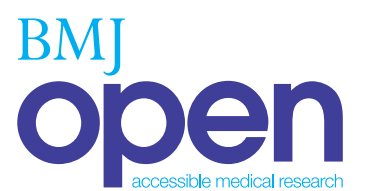

\title{
Transferring responsibility and accountability in maternity care: clinicians defining their boundaries of practice in relation to clinical handover
}

\author{
Georgiana S M Chin, ${ }^{1}$ Narelle Warren, ${ }^{2}$ Louise Kornman, ${ }^{3}$ Peter Cameron ${ }^{1}$
}

To cite: Chin GSM, Warren N, Kornman L, et al. Transferring responsibility and accountability in maternity care: clinicians defining their boundaries of practice in relation to clinical handover. BMJ Open 2012;2:e000734

doi:10.1136/bmjopen-2011000734

- Prepublication history for this paper are available online. To view these files please visit the journal online (http://dx.doi.org/10.1136/ bmjopen-2011-000734).

Received 24 January 2012 Accepted 12 July 2012

This final article is available for use under the terms of the Creative Commons Attribution Non-Commercial 2.0 Licence; see http://bmjopen.bmj.com

\section{${ }^{1}$ Centre for Research} Excellence in Patient Safety, Department of Epidemiology and Preventative Medicine,

The Alfred Centre, Monash University, Melbourne, Victoria, Australia

${ }^{2}$ School of Psychology and Psychiatry, Monash University, Caulfield East, Victoria, Australia ${ }^{3}$ Department of Obstetrics and Gynaecology, University of Melbourne, Parkville, Victoria, Australia

Correspondence to Dr Georgiana S M Chin; Georgiana.Chin@monash. edu.au, Georgiana.Chin@ thewomens.org.au

\section{ABSTRACT}

Objective: This exploratory study reports on maternity clinicians' perceptions of transfer of their responsibility and accountability for patients in relation to clinical handover with particular focus transfers of care in birth suite.

Design: A qualitative study of semistructured interviews and focus groups of maternity clinicians was undertaken in 2007. De-indentified data were transcribed and coded using the constant comparative method. Multiple themes emerged but only those related to responsibility and accountability are reported in this paper.

Setting: One tertiary Australian maternity hospital. Participants: Maternity care midwives, nurses (neonatal, mental health, bed managers) and doctors (obstetric, neontatology, anaesthetics, internal medicine, psychiatry). Primary outcome measures: Primary outcome measures were the perceptions of clinicians of maternity clinical handover.

Results: The majority of participants did not automatically connect maternity handover with the transfer of responsibility and accountability. Once introduced to this concept, they agreed that it was one of the roles of clinical handover. They spoke of complete transfer, shared and ongoing responsibility and accountability. When clinicians had direct involvement or extensive clinical knowledge of the patient, blurring of transition of responsibility and accountability sometimes occurred. A lack of 'ownership' of a patient and their problems were seen to result in confusion about who was to address the clinical issues of the patient. Personal choice of ongoing responsibility and accountability past the handover communication were described. This enabled the off-going person to rectify an inadequate handover or assist in an emergency when duty clinicians were unavailable.

Conclusions: There is a clear lack of consensus about the transition of responsibility and accountability-this should be explicit at the handover. It is important that on each shift and new workplace environment clinicians agree upon primary role definitions, responsibilities and accountabilities for patients. To provide system resilience, secondary responsibilities may be allocated as required.

\section{ARTICLE SUMMARY}

Article focus

- This paper reports on maternity clinicians' perceptions of transfer of their responsibility and accountability for patients with a particular focus on transfers of care within the birth suite. These perceptions may be used to inform further improvements and research in this area.

Key messages

- Despite the recognised definition of clinical, handover, there is still a clear lack of consensus among clinicians about when responsibility and accountability for patients is transferred at clinical handover. This may lead to confusion and not addressing patient problems in a timely manner.

- The transfer of responsibility and accountability for patients should be made clear at the time of handover. This includes a clear definition and allocation of primary roles, responsibilities and accountabilities for team members.

- Secondary responsibility may be allocated to appropriately experienced people to enable flexibility to cover emergency situations when clinicians are unavailable.

\section{BACKGROUND}

Maternity care involves the care of pregnant women through the transition into motherhood. Complexity of care throughout the antenatal to postnatal period is determined by coexistent medical and surgical conditions and psychosocial issues that may or may not be related to pregnancy. The complexity of a patient's clinical state defines the diversity and number of clinicians involved in the care of the patient. ${ }^{1-3}$ Multiple transitions of care are expected to take place throughout 


\section{ARTICLE SUMMARY}

Strengths and limitations of this study

- A particular strength of this study was the involvement of clinicians working in the maternity unit in the hospital to provide contextual knowledge and experience in the conceptualisation, design, data collection and data analysis. This provided useful local knowledge.

- There was the potential of the interview and focus group facilitator's working relationship with the participants to create bias in responses. This was minimised in conducting research outside of the immediate working environment and at times when the clinician was not identified to be on clinical duty. By drawing these boundaries it facilitated engagement of the participants with the interviewer in their research rather than clinical role.

- The study of clinicians of different specialities and seniority who undertake maternity care in one hospital enabled triangulation of data was also one of the strengths in this study. This provided interesting insights of perceptions of roles, relationships and work within the maternity team.

- By localising the research to one study site limits the scope to one hospital. Other studies are required to see whether these findings are also seen in other similar working environments.

- Technical issues resulted in the absence of audio-recording of a small part of the interviews and focus groups which may have had an impact on retaining more nuanced information. However, the use of note-taking at the time improved the ability to retain important information which was able to be used for triangulation with other transcripts.

the maternity care of patients which requires clinical handovers between clinicians. ${ }^{4}$ This includes the birth suite where in labour a woman's care may straddle more than one rostered shift of clinicians and involve multiple specialities dependent on her needs. ${ }^{5-7}$

Clinical handover has been defined as the transfer of responsibility and accountability between one or more clinicians on a temporary or permanent basis. ${ }^{8}$ This official definition of clinical handover exists in national guidelines and more specifically in guidelines of professional obstetric practice. $^{8}{ }^{9}$ However, a specific legal determination for clinical handover is not mentioned in Australian law. Lack of communication in maternity care resulting in an adverse outcome has appeared in a previous Australian legal case. Failure of communication about a previous classical caesarean, which carried an increased risk of uterine rupture, resulted in long-term neurological disability for the affected child following an emergency delivery following uterine rupture in a subsequent pregnancy. ${ }^{10}{ }^{11}$ Although clinical handover was not specifically mentioned in those terms, the concepts of the legal aspects of responsibility and accountability for the handover of relevant information (in this case, written documentation), involvement of the patient in the handover through informing them of relevant information to communicate to health professionals to assist in future decision making and the role in the recipient of the handover and the organisation where they work are illustrated by this case. Information transfer is implicit within the official definition of clinical handover and that the information is understood by the recipients. ${ }^{12}$ Following on from this, liability was attributed to both the first hospital (where the first caesarean section occurred) and the second hospital (where the affected baby was born) for contributing in the communication breakdown that resulted in this adverse event. ${ }^{10} 11$

Transfer of responsibility, accountability and information is part of a subset of reported functions and roles of this process in the literature. ${ }^{13}$ The unambiguous transfer of responsibility has been observed as one of the handover strategies to improve team coordination in work settings with high consequences for failure. ${ }^{14}$ Blurring of boundaries of responsibility have been linked to communication-related healthcare incidents, particularly associated with increasing numbers of clinicians and specialities involved in same patient care. ${ }^{15}$ The introduction of standardised handover protocols that involve clarification of time and/or designation of responsibility have been suggested as improvement strategies with varying success regarding unambiguous transfer of responsibility and accountability. ${ }^{15-17}$

Apart from the official definition, other functions that have been reported include induction to the profession (eg, language, practices, enculturalisation), education, safety through cross-checking (eg, medication and charts), discussion on management issues, organisation, patient management, and discharge planning and socialisation (eg, social/emotional support and debriefing, stress relief, light hearted episodes) ${ }^{18-22}$ Problems with this process have been associated with morbidity and mortality. ${ }^{23-27}$ Inadequate handover has also been associated with problems with lack of coordinated team work (eg, duplication of tests). ${ }^{26} 2829$

Primary maternity care in Australia is undertaken by midwives, and doctors, including general practitioner (GP) obstetricians, obstetricians and obstetric trainee doctors under supervision. This is divided into public and privately funded healthcare. ${ }^{30}$

Although the official definition of clinical handover is assumed to be understood and accepted by clinicians involved, less is known whether this is truly the case.

\section{OBJECTIVE}

This paper reports on maternity clinicians' perceptions of transfer of their responsibility and accountability for patients with a particular focus on transfers of care within the birth suite. These perceptions may be used to inform further improvements and research in this area.

\section{DESIGN}

A qualitative study of clinician perceptions of maternity clinical handover was undertaken in 2007.

Written and verbal information was provided and clinicians provided informed verbal consent before participation. Semistructured interviews (27 face-to-face and 6 written) and 18 focus groups took place facilitated by 
the first author. This format enabled exploration of themes that were raised at that time and enabled testing of themes that had developed in previous interviews and focus groups. Face-to-face interviews and focus groups were audio-recorded and transcribed.

Data were de-identified and themes were coded by the first author using the constant comparative method, ${ }^{31}$ which is founded on a grounded theory methodological approach. ${ }^{32}$ Themes were identified from transcripts and its presence was searched for in other interviews. A sample of three interviews and two focus group transcripts was independently coded by the second author for inter-reliability testing. When coding for presence and lack of presence of first author identified themes, a $74.04 \%$ agreement with a kappa of 0.34 on codes between the two authors was shown. Themes were discussed with the other members of the team. Where ambiguity or disagreement was identified, the transcripts were revisited and consensus achieved. NVivo software was used for data management.

The use of triangulation within focus groups and other transcripts, clarification of points during data collection with the participants and multidisciplinary review of coded data by other members of the research team provided rigour to this study. Further information about the background of researchers is outlined below.

The theme of responsibility and accountability was one of multiple themes that emerged from the analysis, and is reported in this paper.

Ethics for this study was provided by The Royal Women's Hospital Research and Human Research Ethics Committee (Project approval number 06/34) and Monash University Human Research Ethics Committee (Project approval number 2010000497). It complies with the Declaration of Helsinki. ${ }^{33}$

\section{SETTING}

Clinicians who were engaged in the provision of maternity healthcare at one Australian tertiary maternity hospital were invited to participate in this study. This metropolitan, standalone women's hospital provided care for 5000 births per annum at that time. It was a major referral, teaching and research centre in Australia. It was able to provide maternity and newborn services to complex pregnancies including those requiring the provision for ventilation for babies following birth for that Australian state as well as for low-risk pregnancies from the local area.

In this hospital primary public maternity healthcare was undertaken by a combination of midwives, GP obstetricians and obstetric doctors (obstetricians and trainees) for antenatal outpatient care. In the intrapartum and hospital admission (antenatal and postnatal) setting, this primary maternity care was undertaken by midwives and obstetric doctors (obstetricians and trainees). Involvement by other specialities was based on the medical and psychosocial needs of the patient.
Patients identified as lower maternity care risk were managed within one of two lower maternity risk care teams or within a family birth centre environment. In this group, care was predominantly undertaken by midwives with support from obstetric doctors, specialities and allied healthcare workers only as required. Those identified as high maternity care risk were managed in one of two maternal-fetal medicine units or smaller subspeciality units (eg, fetal management unit, diabetes unit, recurrent miscarriage unit, cervical surveillance unit, multiple pregnancy unit, women with physical and/or intellectual disability in pregnancy unit, drugs and alcohol in pregnancy unit, adolescent pregnancies unit). Birth was facilitated by midwives predominantly if they were spontaneous births and obstetric doctors if assisted vaginal birth or caesarean section were required. If interventions such as augmentation or continuous cardiotocographic monitoring were required and if patients were identified as increased obstetric, medical or psychosocial risk, obstetric doctors undertook primary management of these women.

Although not a major focus in this study, some of the clinicians also worked within the private healthcare system. Private maternity healthcare in Australia is predominantly undertaken by obstetricians. Private obstetricians practise either as solo practitioners, in an associateship (one or more obstetricians practising alongside each other but independently accountable from one another) or within a group practice where on-call cover is shared between the group of obstetricians. Referrals are made to other specialists as the needs arise. Solo obstetricians and those in associateship may also choose to share the work with other obstetricians for some on-call and when they are on leave from their practice. Within the private hospital environment, midwives and other specialists (as required) also provide care for the patient. However in this context, the obstetrician or their appointed covering obstetrician assumes obstetric responsibility and accountability for the patient.

To a lesser extent, some women choose to have private care with independent practising midwives providing homebirth support, which may be supported by a GP obstetrician. Private planned homebirths have the opportunity for transfer to the public healthcare system when increased obstetric risk and/or complications arise.

Official clinical handover in this public and private maternity care environment was predominantly unidisciplinary for shift and in private healthcare, on-call/ leave cover. In the public hospital where this study was based, maternity shift handover was face-to-face. Multidisciplinary presence at some public maternity shift handovers occurred at this time with obstetric doctors giving handover at these meeting corresponding to obstetric doctor shift change. Clinicians of differing seniority were present at these handovers. There were two separate birth suites, one family birth unit, two designated postnatal and one designated antenatal ward in operation at the time of this study in the hospital. The 
birth suites contained labouring and birthing patients, those requiring more one-to-one care for acute antenatal and postnatal conditions and for some emergency antenatal assessments. The family birth unit provided some low-risk maternity patients antenatal, intrapartum and postnatal care predominantly by a core group of midwives with support from other clinicians as required. They practised group shift midwifery handover away from the patient. Subsequent one-to-one midwife handover was performed later between off-coming and on-coming midwives. One birth suite which was designated to have lower maternity risk patients (but also had higher risk patients when there was high bed occupancy) had a policy in encouraging this one-to-one handover to occur at the bedside in the presence of the patient.

This description of the maternity healthcare services in the study area has been based on unpublished data from transcriptions in this study, personal experience and knowledge working in this environment of two of the obstetricians in the study group and literature describing provision of Australian maternity healthcare. ${ }^{30}$

\section{PARTICIPANTS}

Participants included 47 midwives, 30 obstetric doctors, neonatal clinicians ( 14 doctors and 8 nurses), 8 anaesthetists, physicians (2 internal medicine and 3 haematology), mental health clinicians (3 doctors and 1 nurse) and 2 nursing bed managers.

Focus groups were purposely in the majority unidisciplinary, of similar seniority or populated by clinicians of the same ward. This enabled participants to be able to freely speak about perceptions minimising the influence of dominance from clinicians of higher seniority or from other specialisations. Focus groups of similar disciplines or work location enabled testing within the group of similar experiences from a similar perspective.

\section{REFLEXIVITY}

The first author who conducted the interviews and focus groups is an obstetrician who undertook specialist training and then consultant work in the study hospital. At the time of the data collection she was a newly qualified consultant (less than 2 years from specialisation) so had an ability to engage at either a more junior or senior level with the participants.

Observational research by clinicians in their working environment exists in published literature. Particularly in ethnographic research there are examples of clinicians undertaking observational work while also undertaking clinical duties in the same area. ${ }^{21} 34$ The impetus for our group studying maternity handover had its roots within the earlier clinical work of the first author. Having a local cultural and technological awareness of the context of study data through the first author's personal working experience in this hospital facilitated: (i) a native understanding of the language used by the participants; (ii) an exploration of concepts raised within the interviews and focus groups; and (iii) provided a conceptual basis for the development of understanding of this area from comparison and testing of perceptions provided by participants in this study.

However, in this study all interviews and focus groups were done outside the immediate work environment and at times where the researcher was identified as being outside clinical duty. This was to encourage the participants to engage with the interviewer as much as possible in her research role rather than her clinical role. This definition between working and clinical roles was also to decrease the element of bias that may occur in blurring of this boundary. In this study, frankness displayed in the transcripts particularly from other specialities and junior obstetric doctors reflected the ability of participants to focus on the interviewer's research rather than the clinical relationship to the participant with the interviewer.

Other authors included: (i) medical anthropologist with an interest in patient-provider interactions who also had recent experience as a maternity patient in the public healthcare system; (ii) senior obstetrician who provided a clinical, administration and education role in the hospital where the study took place; and (iii) emergency physician with a background in quality and safety research. These researchers and clinicians did not undertake any of the interviews but were involved in the conceptualisation, design, analysis and dissemination of this research. They provided a multidisciplinary and healthcare consumer input into this study. Having multiple viewpoints provided rigour to the study, analysis and reporting.

\section{PRIMARY OUTCOME MEASURES}

Participants' perceptions of current practice, setting, expectations and future improvements pertaining to maternity clinical handover.

\section{RESULTS}

In most cases, participants did not spontaneously connect the transfer of responsibility and accountability as a function or role of clinical handover. Clinicians spontaneously reported the primary function of handover being information transfer, patient care and/or continuity of care in all but one interview and one focus group. In the one focus group where it was not spontaneously reported, logistical management of resources, beds and staff and, accountability were thought immediately associated with the functions and roles of handover. In one interview, the transfer of responsibility and accountability was spontaneously reported as the primary role of handover above any other function and role.

In total were 7 clinicians in this study who spontaneously identified the transfer of responsibility and/or accountability as one of the roles or functions of handover. However, when asked specifically about this function, the other clinicians agreed that it was indeed connected to handover. Once introduced to this concept, 
Box 1 Example quotations about transfer of responsibility and accountability

Complete transfer of responsibility and accountability

So I'm the one looking after her, I decide to stay, yeah, and some other midwife comes in, well you're right, there's never anything officially said, but, I would assume I'm still the one responsible and if that happens with me coming into a room, I would assume that they were responsible...Well, till they leave.

(Senior midwife)

[describing when responsibility and accountability has been transferred] When you have communicated your concerns about the patient, when you've communicated any, any issue, any management or issues that have come up whether it be from the patient perspective or from the clinician's perspective and when you feel that when you're satisfied that the team that's taking over um, understands what your proposed plan of management was, whether they agree with it or, or not um, and have enough information to be able to make sound clinical judgements with what they're going to do.

(Junior obstetric doctor (Level 2 Registrar))

When the handover's completed...And if we don't know about a patient, then it's hard to take responsibility for what goes on, um, and equally can't provide care.

(Consultant obstetrician)

...You are accountable for the information transferred as well as received...You can't literally handover all sixty babies on the floor of nursery so there will be anticipated problems that may occur in the next shift, so from a reviewer perspective you want to handover what has been done and what problems that you might anticipate in nursery or in obstetric deliveries. The role of handover is for that safe anticipation of a patient's journey.

(Consultant paediatrician)

Shared responsibility and accountability

There's a couple of hours where that's not clear because you're both there and although we have um, you know, like an education time after handover and the afternoon staff go to that and so technically the morning staff still cover that woman until whenever. In my own practice if I get handed to at two thirty, before I do anything um, I make sure that I've seen what I'm responsible for and if I'm able to I go to education sessions then but I like to look at, you know, exactly what I'm taking up before I let that other person go.

(Midwife)

I believe I am transferring partial responsibility for the patient because that personal responsibility has not passed. Occasionally I continue to take part in the management especially if I am MFM [Maternal Fetal Medicine] Registrar or know her from the ward, I know that I will be looking after this patient postpartum and I always ask what has happened to her. But I think my responsibility, if I finished night duty, I hand over patient to the morning staff and continuity to the staff. I still have responsibility for what I have done, what I made. It is difficult what you mean, responsibility on a legal point of view, mental point of view, moral point of view?... Legally I feel responsibility but I hand over, morally I feel responsible as well, can I say shared responsibility?

(Junior obstetric doctor (registrar))
Ongoing responsibility and accountability

[on the timing of transferring responsibility and accountability] When you decide to go home. Sometimes you'll stay until four o'clock [thirty minutes after the official end of morning shift] and there's still no birth and you're thinking it's time I went and you walk.

(Senior midwife)

Definitely especially if I have been with the patient full time and this patient is in 2nd stage is very close to delivery after 20 hours, especially labour related handover and I handover someone who is almost fully dilated, occasionally I feel that I carry full responsibility and next day I found something had happened, it was a difficult delivery, I feel responsible that I should have partly done something for this patient.

(Junior obstetric doctor (registrar))

[speaking about being paged after handover] But if they have told you that there is no one else around, the patient is having late decels [signifying non-reassuring fetal monitoring], I guess you can't do much, at least you have taken the call and given them some basic instructions and what to do and although they are basic and the midwives may have already done them first it always helps to re-iterate things.

(Junior obstetric doctor (Level 2 registrar))

I guess for me, within our department, it is if you are rostered on you are responsible, and when you are not on that responsibility is transferred to the next person. It's really that time base thing, although there is often, often do carry that responsibility say if I have got a patient that I have been particularly involved with needing to make sure she is okay even if I am not on. And that probably provides more confusion, 'cause what's [Doctor's Name] ringing when she's not on.

(Consultant physician (haematologist))

they spoke about the transfer of responsibility and accountability in terms of complete transfer as well as shared and ongoing personal responsibility and accountability (box 1).

\section{Complete transfer of responsibility and accountability}

Complete transfer of care was marked by some as corresponding to the end of the handover communication (which may be part of an organisational protocol), by rostered time of duty, actual commencement of work or by the clinician leaving the workplace at the end of the shift. Although it was highlighted by a few that this happens regardless of the communication, some clinicians stressed that they were responsible and accountable for delivering the information required for a safe transition of care and that it be understood by the on-coming team.

Midwives had first a group ward or birth suite handover followed by allocations of patients and finally an individual one-to-one handover. Some midwives, specifically mentioned that the complete transfer of care was marked by the end of the one-to-one handover with the off-coming midwife of their allocated patients. 
The importance of seniority was also mentioned by some clinicians. This was discussed in terms of responsibility and accountability being transferred once handover was given to a clinician of similar or of greater seniority than the handing-over clinician.

\section{Shared responsibility and accountability}

For some clinicians, responsibility and accountability was felt not to be handed over completely at the clinical handover communication. At times where there was a regular overlap of rostered shift time for the off-coming and on-coming clinicians (30 min for the obstetric doctors and up to $2 \mathrm{~h}$ in the case of midwives handing over in the middle of the day), this was seen either as a period of shared or blurred responsibility and accountability until the off-coming clinician went home. Having this overlap in rostered shifts allowed for handover communication to take place and for the midwives, the opportunity for a group to have education sessions while being covered by others on the ward.

Some clinicians still felt ties to the patient due to their own extensive personal clinical knowledge of the patient, their role within the continuity of care of the patient in the hospital or their extensive direct involvement in the patient's care. One clinician even expressed this as the division of legal and moral responsibility for a patient's care. In this case, both can be handed over at a shift change, but this clinician felt that they morally had ongoing responsibility for a patient.

\section{Ongoing personal responsibility and accountability}

Some clinicians felt an ongoing personal responsibility and accountability for patients despite having given a handover to the on-coming staff. Examples of this were in the case of incomplete handover and personal professional attachment to the patient's clinical well-being and care. This was acknowledged by one clinician, a physician, as potentially causing confusion.

Some clinicians felt responsible and accountable for the information that they gave at handover including ensuring tasks that were handed over were understood. Some also felt that if after handover the on-coming clinician was unavailable for an emergency, the off-coming clinician had a duty of care to help if they could if paged. If the clinician did not handover everything required for assumed patient care, some clinicians felt that they were still responsible and accountable for the patient until they handed over the information; even if this meant calling up the ward, after they had left, to handover.

One clinician noted that despite handing over care, if the outcome of a labour was a difficult delivery following the handover, they felt responsible for the outcome. This illustrated the ambiguous relationship between management decisions prior to handover that potentially result in adverse outcomes following handover. Although the transfer of information at this time point can be defined, less obvious to the clinician was where their responsibility and accountability lies in the continuum of care resulting in the health of the mother and baby following birth. This could happen whether or not the outcome was within the clinician's control.

Both midwives and obstetric doctors spoke of ongoing responsibility and accountability of patients in labour following an official handover. This occurred in the public and privately insured healthcare systems. Some of these clinicians who had direct care of the patient during this time chose and felt responsible and accountable for the patient until after the birth had been completed; even if this meant working past the end of their rostered shift. This enabled continuity of clinician throughout this key time of transition to motherhood. This practice was acknowledged as an accepted but not necessarily an expected practice by other clinicians.

One clinician in particular reflected on how it was difficult for clinicians to leave the workplace and that learning to hand over care and leave was important for clinician longevity within the workforce.

\section{Defining who is responsible}

A few clinicians commented about who was responsible for patient care (box 2). They spoke about defining work and responsibilities within the on-coming team.

\section{Box 2 Example quotations about defining who is} responsible

\section{Defining roles and responsibilities}

...t's now, l'm leaving, and it's not your responsibility. So I guess it's also depending on how workload is shared I think you do need to define who will be responsible for checking progress, or a result or reviewing a patient, and that's a shared responsibility.

(Consultant physician (haematologist))

..Very recent times we have moved to having very set times that you have of responsibility for something, which makes it much easier for someone to just "oh it's really my responsibility today", in to move on, and I think that you see in all levels, I mean you see it where residents, maybe less so with the registrars, because I think a lot of them are a little bit more aware of the fact that they've made his, they have made the transition, they have the responsibility...But the problem is everybody kind of, reaches that point where they're not really involved, and is there's no single person who has that ownership over the patient for the problems that arise, so, I don't know how that's going to change though.

(Junior anaesthetic doctor (Senior registrar/fellow))

Seniority defining responsibility

Sure, the minute l'm assuming the handing over is to a team who are starting a shift, so within that team there will be a pecking order of accountability and responsibility, and I would therefore assume that if it's the morning handover and your handing over from the night shift to the morning shift it will be a registrar, nurse manager, the relevant midwives who will be each - the departing person will be replaced by an incoming person who will have the same level of accountability and responsibility.

(Consultant obstetrician) 
They also described a person's clinical area and expertise defining their work and responsibilities. One anaesthetic doctor described it is a matter of ownership: responsibility and accountability for a patient being equated with the ownership of the care of the patient and associated issues.

One junior obstetric doctor felt that the allocation of responsibility was to the most senior person on the shift: the consultant. He felt that although the consultant obstetrician was responsible, he saw that person as separate from the team and the role of the consultant was to provide expert opinion at a distance.

\section{DISCUSSION}

Transition of responsibility and accountability for patients is recognised as an essential part of clinical handover. ${ }^{8}$ However, within this study there was a lack of agreement between clinicians when this took place.

Some clinicians in this study described the transition of responsibility and accountability for patients as occurring once communication of information needed for the safe transfer had taken place. Some also recognised that this did not occur until there was acknowledgement or a sign of understanding of this information from the on-coming clinicians. ${ }^{12}$

Other clinicians took a more temporal and physical approach to handover by describing it in terms of the rostered shift or when a clinician physically left the work environment. Shift work is something that is well understood by those in the nursing and midwifery profession with handover being seen by some as almost a ritualised practice. $^{21}$ However, this is a newer concept to the medical profession with the transition from the historical personal continuity of care to the development of the modern 'safe hours' shift work phenomenon. ${ }^{35}$ The establishment of the modern 'safe hours' for medical staff recognises that clinicians do not work at their best or even safely when fatigued. Issues with cognition, response time, attention and increased rates of errors are associated with the traditional longer shifts and on-call duty. ${ }^{36-39}$ With the advent of shift work for medical staff, it has become imperative for doctors to understand when it is time to hand over care of a patient and to become more familiar with team communication. ${ }^{35}$

Some clinicians in this study experienced confusion or blurring of boundaries of responsibility and accountability. This is was when clinicians still felt responsibility and accountability for patients once a handover had taken place. There were times when this ambiguity in responsibility and accountability was observed to be beneficial. Examples given by participants were when staff completing a shift called back to add or correct information from an inadequate handover or responding to an emergency when on-coming staff were unavailable. In these cases, the impression of ongoing responsibility and accountability provides system resilience; in other words an informal back-up strategy when there is a system issue. ${ }^{40}$
However, confusion or blurring of responsibility and accountability following a handover can lead to problems. Role confusion, uncertainty of allocation of work and identification of the appropriate person for reporting and inquiry for the status and management for patients may be the result of such ambiguity which may lead to clinical incidents. ${ }^{15} 41$ There is also the potential for significant psychological impact on a clinician feeling ongoing responsibility and accountability, real or misinterpreted, for an unexpected, unwanted and/or adverse outcome. It may result in the clinician changing their behaviour, questioning whether to continue to work in the profession and/or negatively impact on a clinician's psychological well-being. ${ }^{42}{ }^{43}$ It is important that clinicians not to feel falsely responsible or accountable in such cases and receive the appropriate support and counselling as required. However, such support may not routinely occur. ${ }^{42} 43$

Conversely, blurring boundaries of responsibility and accountability may result in no one taking responsibility. ${ }^{15}$ As one clinician in our study described it, lack of 'ownership' reflects the potential for no clinician taking on the role or responsibility for addressing patient problems. Although team training in maternity services is beginning to show some improved clinical outcomes, ${ }^{44}$ such elements as role definition, allocation of work, responsibility and accountability should be agreed upon and established each shift and/or handover, as well as a topic for interdisciplinary education. ${ }^{45}$

\section{CONCLUSION}

This study has shown that there is a clear lack of consensus when responsibility and accountability are transferred. When commencing work within a new work environment or shift it should be clarified when responsibility and accountability are passed on. There should also be a clear definition and allocation of primary roles, responsibilities and accountabilities for team members. This is a keystone to safe clinical care.

Secondary responsibilities may also be allocated to appropriately experienced people at the end of a shift on an ad hoc basis to enable flexibility in the system to cover emergency situations.

\section{STRENGTHS AND LIMITATIONS}

A strength of this study was the involvement of clinicians working in the maternity unit in the hospital to provide contextual knowledge and experience in the conceptualisation, design, data collection and data analysis. This provided useful local knowledge.

There was the potential of the interview and focus group facilitator's working relationship with the participants to create bias in responses. This was minimised by interviewing outside the immediate working environment and at times when the clinician was not identified to be on clinical duty. By drawing these boundaries it 
facilitated engagement of the participants with the interviewer in their research rather than clinical role.

The study of clinicians of different specialities and seniority who undertake maternity care in one hospital enabled triangulation of data was also one of the strengths in this study. This provided interesting insights of perceptions of roles, relationships and work within the maternity team. However, by localising the research to one study site limited the scope to one hospital. Other studies are required to see whether these findings are also seen in other similar working environments.

Technical issues resulted in the absence of audiorecording of a small part of the interviews and focus groups, which may have had an impact on retaining more nuanced information. However, the use of notetaking at the time improved the ability to retain important information which was able to be used for triangulation with other transcripts.

Acknowledgements We acknowledge the clinicians who kindly gave their time to participate in this study.

Contributors GSMC, LK and PC participated in the conception and design of this study. GSMC conducted the interviews and focus groups. GSMC, LK, NW and PC participated in the analysis and interpretation of data, with significant contribution to analysis by GSMC and NW. All four authors participated in the drafting and approval of the final approval of the manuscript.

Competing interests All authors have completed the Unified Competing Interest form at www.icmje.org/coi_disclosure.pdf (available on request from the corresponding author) and declare: GSMC was funded by an Australian Postgraduate Award (APA) as part of her doctoral research; GSMC and LK were employed as clinicians at the study site at the time of the study; no other relationships or activities that could appear to have influenced the submitted work.

Provenance and peer review Not commissioned; externally peer reviewed.

Data sharing statement Additional unpublished data from the study are available to the authors of this paper for the purposes of further analysis and publication in preparation for completion of the $\mathrm{PhD}$ thesis of the first author.

\section{REFERENCES}

1. National Collaborating Centre for Women's and Children's Health. NICE Clinical Guideline No. 62. Antenatal care: Routine care for the healthy pregnant woman. London: RCOG Press, 2008.

2. National Collaborating Centre for Women's and Children's Health. Nice Clinical Guideline No. 110. Pregnancy and complex social factors: a model for service provision for pregnant women with complex social factors. London: National Institute for Health and Clinical Excellence, 2010.

3. Cantwell RC, Mahmood TA. Good Practice No. 14. Management of women with mental health issues during pregnancy and the postnatal period. UK: Royal College of Obstetricians and Gynaecologists, 2011.

4. Hatten-Masterson SJ, Griffiths ML. SHARED maternity care: enhancing clinical communication in a private hospital setting. Med $J$ Aust 2009;190(11 Suppl):S150-S1.

5. Berridge E-J, Mackintosh NJ, Freeth DS. Supporting patient safety: examining communication within delivery suite teams through contrasting approaches to research observation. Midwifery 2010;26:512-19.

6. Siassakos D, Draycott TJ, Crofts JF, et al. More to teamwork than knowledge, skill and attitude: General obstetrics. BJOG 2010;117:1262-9.

7. Ottewill M, Urben J, Elson D. Safe hand-over: safe care. Midwives 2007;10:508-9.

8. Australian Medical Association (AMA). Safe handover: safe patients. Guidance on clinical handover for clinicians and managers. The Australian Medical Association Limited. Canberra, Australia. 2006.

9. The Royal Australian and New Zealand College of Obstetricians and Gynaecologists. Clinical Handover (WPI-19). 2011.
10. Flannigan AC, Hogan-Ross K. Australia: Obstetrics and Women's Health. Summary of recent cases. DLA Piper [serial on the Internet]. 7 April 2010. http://www.mondaq.com/australia/article.asp? articleid $=97670$

11. Supreme Court of New South Wales-Court of Appeal. [2008] NSWCA 335. 2008.

12. Jorm C, ledema R. Innovative approaches to enhancing clinical handover. Paris, France: International Forum on Quality and Safety in Health Care, 2008.

13. Patterson ES, Wears RL. Patient handoffs: standardized and reliable measurement tools remain elusive. Jt Comm J Qual Patient Saf 2010;36:52-61.

14. Patterson E, Roth E, Woods D, et al. Handoff strategies in settings with high consquences for failure: lessons for health care operations. Int J Qual Health Care 2004;16:125-32.

15. Williams RG, Silverman R, Schwind C, et al. Surgeon information transfer and communication. Factors affecting quality and efficiency in inpatient care. Ann Surg 2007;245:159-69.

16. Catchpole KR, de Leval MR, McEwan A, et al. Patient handover from surgery to intensive care: using Formula 1 pit-stop and aviation models to improve safety and quality. Pediat Anae 2007;17:470-778.

17. Wayne JD, Tyagi R, Reinhardt G, et al. Simple standardized patient handoff system that increases accuracy and completeness. $J$ Surg Edu 2008;65:476-85.

18. Kerr MP. A qualitative study of shift handover practice and function from a socio-technical perspective. J Adv Nurs 2002;37:125-34.

19. Lally $S$. An investigation into the functions of nurses' communication at the inter-shift handover. J Nurs Manag 1999;7:29-36.

20. Sexton A, Chan C, Elliott M, et al. Nursing handovers: do we really need them? J Nurs Manag 2004;12:37-42.

21. Strange F. Handover: an ethnographic study of ritual in nursing practice. Intensive Crit Care Nurs 1996;12:106-12.

22. Yonge $O$. Shift report: a ritual play on a residential adolescent psychiatric unit. J Psychiatr Ment Health Nurs 2008;15:45-51.

23. Lewis G. The Confidential Enquiry into Maternal and Child Health (CEMACH). Saving Mothers' Lives: reviewing maternal deaths to make motherhood safer-2003-2005. The Seventh Report on Confidential Enquiries into Maternal Deaths in the United Kingdom. London: CEMACH, 2007.

24. Australian Institute of Health \& Welfare, Australian Commission on Safety and Quality in Health Care. Sentinel events in Australian public hospitals 2004-05. Cat. No. HSE. 51. Canberra: AIHW, 2007.

25. Jorm CM, White S, Kaneen T. Clinical handover: critical communications. Med J Aust 2009;190(11 Suppl):S108-9.

26. O'Byrne W 3rd., Weavind L, Selby J. The science and economics of improving clinical communication. Anesthesiol Clin 2008;26:729-44.

27. Boseley S. NHS hospital in spotlight after womens' postnatal deaths. The Guardian, 201129 September 2011; Sect. 11.

28. Sabir N, Yentis SM, Holdcroft A. A national survey of obstetric anaesthetic handovers. Anaesthesia 2006;61:376-80.

29. Bomba D, Prakash R. A description of handover processes in an Australian public hospital. Aust Health Rev 2005;29:68-79.

30. Australian Government National Health and Medical Research Council. National guidance on collaborative maternity care. Commonwealth of Australia, Canberra, Australia. 2010.

31. Markovic M. Analysing qualitative data: health care experiences of women with gynaecological cancer. Field Methods 2006;18:413-29.

32. Corbin J, Strauss A. Basics of qualitative research. Techniques and procedures for developing Grounded Theory. 3rd edn. Sage Publications, Inc., Thousand Oaks, California, United States of America. 2008.

33. Declaration of Helsinki: ethical principals for research involving human subjects. 2008.

34. Manias $E$, Street $A$. The handover: uncovering the hidden practices of nurses. Intens, Crit Care Nurs 2000;16:373-83.

35. Jones GJ, Vanderpump MPJ, Easton M, et al. Achieving compliance with the European Working Time Directive in a large teaching hospital: a strategic approach. Clin Med 2004;4:427-30.

36. Lockley SW, Cronin JW, Evans EE, et al. Effect of reducing interns weekly work hours on sleep and attentional failures. $N$ Engl J Med 2004;351:1829-37.

37. Landrigan CP, Rothschild JM, Cronin JW, et al. Effect of reducing interns' work hours on serious medical errors in intensive care units. N Engl J Med 2004;351:1838-48.

38. Taffinder NJ, McManus IC, Gul Y, et al. Effect of sleep deprivation on surgeons' dexterity on laparoscopy simulator. Lancet 1998;352:1191.

39. Oroton DI, Gruzelir JH. Adverse changes in mood and cognitive performance of house officers after night duty. BMJ 1989;298:21-3.

40. Hollnagel E, Woods DD, Leveson N. Resilience engineering: concepts and precepts. Aldershott, UK: Ashgate, 2006. 
41. Lakasing $\mathrm{L}$, Spencer JAD. Care management problems on labour ward: 5 years' experience of clinical risk management. $J$ Obstet Gynaecol 2002;22:470-6.

42. McCready S, Russell R. A national survey of support and counselling after maternal death. Anaesthesia 2009;64:1211-17.

43. Strote J, Schroeder E, Lemos J, et al. Academic emergency physicians' experiences with patient death. Acad Emerg Med 2011;18:255-60.
44. Reily W, Davis S, Miller K, et al. Diadatic and simulation nontechnical skills team training to improve perinatal patient outcomes in a community hospital. Jt Comm J Qual Patient Saf 2011:37:357-64.

45. Agency for Healthcare Research and Quality. TeamSTEPPS: national implementation. 2011 (cited 2011). http://teamstepps.ahrq gov/about-2cl_3.htm 